[0212-7199 (2007) 24: 8; pp 379-383] ANALES DE MEDICINA INTERNA Copyright (C) 2007 ARAN EDICIONES, S.L.

AN. MED. INTERNA (Madrid) Vol. 24, N. ${ }^{\circ} 8$, pp. 379-383, 2007

\section{Análisis transversal de la insuficiencia cardiaca en pacientes de un Servicio de Medicina Interna de un hospital de tercer nivel de área mixta (rural y urbana). Parte III: análisis de mortalidad (Tercera de tres partes)}

\author{
S. CINZA SANJURJO, A. CABARCOS ORTIZ DE BARRÓN', E. NIETO POL ${ }^{2}$, \\ J. A. TORRE CARBALLADA ${ }^{1}$
}

Fundación Pública de Urgencias Sanitarias 061. ' Servicio de Medicina Interna. Complejo Hospitalario Universitario de Santiago de Compostela.${ }^{2}$ Servicio de Atención Primaria. Centro de Salud Concepción Arenal. Santiago de Compostela. A Coruña
CROSS-SECTIONAL STUDY OF HEART FAILURE OF PATIENTS INTAKED IN AN INTERNAL MEDICINE SERVICE IN THE THIRD LEVEL HOSPITAL IN MIXED AREA. PART III: MORTALITY ANALYSIS

\begin{abstract}
RESUMEN
Objetivos: Analizar las características epidemiológicas de los pacientes fallecidos entre los ingresados por insuficiencia cardiaca.

Material y métodos: Estudio descriptivo transversal, retrospectivo, de pacientes ingresados en el Servicio de Medicina Interna del Hospital Clínico Universitario de Santiago de Compostela a lo largo de 5 años (desde 1999 hasta 2003). Se registraron las variables: sexo, edad, días de ingreso, número de reingresos por insuficiencia cardíaca, motivo de ingreso, hipertensión arterial (HTA) previa, diabetes mellitus (DM) previa, cardiopatía previa, cardiopatía isquémica previa, fibrilación auricular (FA) previa, consumo de beta-bloqueantes previos al ingreso, cifras de presión arterial (PA) al ingreso, realización de ecocardiografía durante el ingreso, fracción de eyección (FE) según ecocardiograma, factor desencadenante, exitus, tratamiento domiciliario al alta. Para el análisis estadístico se emplearon los estadísticos descriptivos cuantitativos y cualitativos que correspondieran; para el análisis bivariante se emplearon chi-cuadrado y " $t$-student".

Resultados: Ingresaron 248 pacientes por insuficiencia cardiaca, de los cuales fallecieron un total de 21 pacientes $(8,6 \%)$. No se observaron diferencias por sexo y la edad media fue superior entre los fallecidos. La mediana de ingreso fue de 5 días, menor que la presentada por la totalidad de la población estudiada, $\mathrm{p}<0,001$. La presencia de HTA $(30,0$ vs. $42,6 \%$, $\mathrm{p}=0,27)$ y cardiopatía isquémica $(30,0 \%$ vs. $27,7 \%, \mathrm{p}=0,82)$ fue similar entre los fallecidos, en comparación con el resto de población. Tampoco se observó mayor HTA en mujeres (16,7 vs. 35,7\%, respectivamente, $\mathrm{p}=$ $0,21)$ ni cardiopatía isquémica en varones $(50,0 \mathrm{vs} .21,4 \%, \mathrm{p}=0,20)$. $\mathrm{La}$ realización de ecocardiogramas fue muy baja entre fallecidos $(21,0 \%)$, muy similar a la del resto de la población $(\mathrm{p}=0,76)$. Aunque se observó mayor prevalencia de depresión de la función sistólica $(80 \mathrm{vs} .41,3 \%)$, esta diferencia no fue estadísticamente significativa $(\mathrm{p}=0,09)$.

Conclusiones: El único factor relacionado con la mortalidad es la edad avanzada de los pacientes. No podemos afirmar que ni sexo ni disfunción sistólica sean marcadores de riesgo de la mortalidad en los pacientes con insuficiencia cardiaca
\end{abstract}

PALABRAS CLAVE: Insuficiencia cardíaca. Epidemiología. Mortalidad.

\begin{abstract}
Objectives: To establish the characteristics of the deceased in intaked patients by heart failure.

Material and methods: A cross-sectional study of the intaked patients in the Internal Medicine Service in the Hospital Clínico Universitario de Santiago de Compostela between 1999 to 2003. The variables analized were: sex, age, days of hospital stay, number of intaked by failure cardiac, reason for admission (guide symptom), hypertension, diabetes mellitus, cardiac disease, fibrillation atrium, previous treatment with beta-blockers, blood pressure in the admission moment, to make echocardiography, disfunction systolic, etiology, deceased, treatment at the end.The statistical analysis was performed with cualitative and cuantitative measures, chi-cuadrado and t-student.

Results: 248 patients were accepted for the study, with the mortality rate rising $8.6 \%$ (21 patients). We did not observed differences between sexes, but the median age in death patients was greater than other patients. The median income was 5 days, letter than study population. The hypertension prevalence (30 vs. $42.6 \%, p=0.27)$ and ischemic cardiopathy (30 vs. $27.7 \%, p=0.82$ ) did not showed differences with the population. The hypertension prevalence in women (16.7vs. $35.7 \%, p=0.21)$ and the ischemic cardiopathy prevalence in men (50 vs. $21.4 \%, p=0.20)$ did not showed differences. It made echocardiography in $21.0 \%$ of death patients, $p=0.76$. The systolic disfunction prevalence was bigger in death patients ( $80 \mathrm{vs.41.3 \% ),} \mathrm{this} \mathrm{difference} \mathrm{was} \mathrm{not} \mathrm{significant} \mathrm{statistically.}$

Conclusions: The older patients showed letter survival. We did not observe any influence of sex or left ventricular systolic function on mortality in patients with heart failure.
\end{abstract}

KEY WORDS: Heart failure. Epidemiology. Mortality.

Cinza Sanjurjo S, Cabarcos Ortiz de Barrón A, Nieto Pol E, Torre Carballada JA. Análisis transversal de la insuficiencia cardiaca en pacientes de un servicio de Medicina Interna de un hospital de tercer nivel de área mixta (rural y urbana). Parte III: análisis de mortalidad (Tercera de tres partes). An Med Interna (Madrid) 2007; 24: 379-383.

\section{INTRODUCCIÓN}

Como ya es conocido, las principales causas de mortalidad en los países desarrollados en general y en España en particu- lar corresponden a las enfermedades cardiovasculares, de forma especial a la cardiopatía isquémica y enfermedad cerebrovascular, ocupando la insuficiencia cardiaca el tercer puesto entre las enfermedades cardiovasculares (1). 
Actualmente es incuestionable el papel que juegan la hipertensión arterial (HTA) y la cardiopatía isquémica (CI) en la etiopatogenia de la insuficiencia cardiaca (IC) (2), ambas patologías crónicas que requieren tratamiento diario, en el cual fármacos como los inhibidores del enzima convertidor de angiotensina (IECA) (3), antagonistas del receptor de angiotensina II (ARA-II) (4) y beta-bloqueantes (5) han demostrado su utilidad y la mayor supervivencia en las tres patologías (HTA, CI e IC), lo que añadido a un envejecimiento poblacional está aumentando progresivamente la prevalencia de IC, que alcanza el $10 \%$ en mayores de 70 años (6), hasta el punto de ser la primera causa de ingreso hospitalario en España (5\% de los ingresos), lo que refleja que la IC, a pesar de presentar mayor supervivencia, limita notablemente la calidad de vida de los pacientes (7).

Son múltiples los datos epidemiológicos que han pretendido relacionarse con el pronóstico de IC, así estudios como Framingham (8), inicialmente, o NHANES (9), posteriormente, mostraban que la mortalidad era superior en varones. En estudios posteriores, analizando posibles factores de confusión demostraron que el valor predictivo de la edad era escaso (10). Actualmente, existe evidencia a favor de que las diferencias observadas entre sexos podrían estar relacionadas con una menor prevalencia de disfunción sistólica en mujeres, al ser menor la etiología isquémica (11).

Otro factor considerado de mal pronóstico fue la existencia de cardiopatía isquémica como factor etiológico de la IC, pero el estudio SOLVD (12) tampoco evidenció diferencias en la mortalidad.

El único factor que realmente se ha asociado con mayor mortalidad desde los estudios Framingham (8) y NHANES (9) fue la edad, de tal forma que a mayor edad, mayor era la mortalidad de los pacientes, llegando a ser 11 veces superior en mayores de 85 años (13).

Tras lo anteriormente expuesto, y disponiendo de una base de datos de pacientes ingresados durante 5 años en un servicio de medicina interna de un hospital de tercer nivel, decidimos plantear el presente estudio que permitiría analizar las características epidemiológicas de los pacientes fallecidos entre los ingresados por insuficiencia cardiaca.

\section{MATERIAL Y MÉTODOS}

Se realizó un estudio descriptivo transversal retrospectivo en el que se revisó la información contenida en los informes de alta, de exitus e historia clínica de los pacientes que ingresaron en el Servicio de Medicina Interna del Hospital Clínico Universitario de Santiago de Compostela entre los años 1999 y 2003 (5 años) con diagnóstico clínico de insuficiencia cardíaca al alta o exitus, según el cumplimiento de criterios de Framingham (14) (Tabla I).

El registro de datos se realizó retrospectivamente a lo largo de los años 2003 y 2004, registrando las variables indicadas en la tabla II para cada paciente. Se definieron la hipertensión arterial (HTA) y la diabetes mellitus según criterios del Joint Nacional Comité VII (JNC-VII) (15) y de la Asociación Americana de Diabetes (16), respectivamente. Se consideró cardiopatía isquémica a la presencia de síntomas, alteraciones electrocardiográficas (ECG) y/o alteraciones angiográficas compatibles con dicha patología en algún ingreso previo o en el mismo ingreso. Se registró el antecedente de fibrilación
TABLA I

CRITERIOS FRAMINGHAM PARA EL DIAGNÓSTICO DE INSUFICIENCIA CARDIACA

\section{Criterios mayores}

Disnea paroxística nocturna u ortopnea

Ingurgitación yugular

Estertores

Cardiomegalia

Edema agudo de pulmón

Galope por tercer tono

Presión Venosa central >16 mmHg

Tiempo de circulación > 25 sg

Reflujo hepatoyugular

\section{Criterios menores}

Edema maleolar

Tos nocturna

Disnea de esfuerzo

Hepatomegalia

Derrame pleural

Reducción de la capacidad vital en un tercio respecto la máxima

Taquicardia (> 120 lat/min)

Pérdida de $>4,5 \mathrm{Kg}$ en 5 días en respuesta al tratamiento (podría ser criterio mayor)

Para diagnóstico de insuficiencia cardíaca se precisan dos criterios mayores o uno mayor y 2 menores. En los criterios menores deben descartarse otras causas.

TABLA II

VARIABLES REGISTRADAS EN EL ESTUDIO

\section{Sexo}

Edad

Días de ingreso

Número de reingresos por insuficiencia cardíaca

Motivo de ingreso

Hipertensión arterial (HTA) previa

Diabetes mellitus (DM) previa

Cardiopatía previa

Cardiopatía isquémica previa

Fibrilación auricular (FA) previa

Consumo de beta-bloqueantes previos al ingreso

Cifras de presión arterial (PA) al ingreso

Realización de ecocardiografía durante el ingreso

Fracción de Eyección (FE) según ecocardiograma

Factor desencadenante

Exitus

Tratamiento domiciliario al alta

auricular (FA) en aquellos casos que presentaran la citada arritmia en el ECG o bien existiera el registro de FA paroxística en la historia del paciente. Se consideró que la fracción de eyección estaba conservada cuando era superior al $45 \%$, deprimida cuando se encontraba entre 30 y $45 \%$ y severamente deprimida cuando era inferior al $30 \%$. Se recogió como factor desencadenante del episodio el que indicó el facultativo en el informe de alta o exitus. 
Para el análisis estadístico se emplearon los estadísticos descriptivos cuantitativos y cualitativos que correspondieran; para el análisis bivariante se emplearon chi-cuadrado y " $t$-student".

\section{RESULTADOS}

A lo largo de los 5 años de estudio (desde 1999 hasta 2003), ingresaron un total de 248 pacientes por insuficiencia cardiaca de los cuales fallecieron durante el ingreso un total de 21 pacientes $(8,6 \%)$. No se observaron diferencias entre sexos en cuanto al fallecimiento $(66,7$ vs. $54,1 \%, \mathrm{p}=0,26)$, manteniendo cierto predominio del sexo femenino, como en la población general $(55,2 \%)$. La mediana de edad fue superior entre los fallecidos (83 años, rango intercuartílico 9,5 vs. 76 años, rango intercuartílico de 12 años, $\mathrm{p}<0,001)$. Se observa cierto predominio, entre los fallecidos, del sexo femenino en mayores de 71 años (100 de mujeres vs. 83,3\% de varones), en cambio no se hallaron diferencias estadísticamente significativas, $\mathrm{p}=0,12$. En la figura 1 se observa la distribución de fallecidos por décadas de edad, en la que se observa que la mayor mortalidad se produce en mayores de 80 años.
La mediana de ingreso para la población total fue de 11 días (rango intercuartílico de 8 días), pero en los pacientes fallecidos fue significativamente menor ( 5 días, rango intercuartílico 7 vs. 11 días, rango intercuartílico 9 días, $\mathrm{p}<0,001$ ).

El análisis de los antecedentes personales no mostró ninguna diferencia en la prevalencia entre fallecidos y no fallecidos de las patologías analizadas: HTA (30 vs. 42,6\%, p =0,27), DM (20 vs. 31,0\%, p =0,30), cualquier cardiopatía previa (80 vs. $81,4 \%, \mathrm{p}=0,88)$, cardiopatía isquémica $(30 \mathrm{vs} .27,7 \%, \mathrm{p}=0,82)$ y fibrilación auricular $(29,5$ vs. $19,0 \%, \mathrm{p}=0,31)$. Se observó una prevalencia similar de HTA entre varones y mujeres (16,7 vs. $35,7 \%$, respectivamente, $\mathrm{p}=0,21$ ) y también fue similar la distribución de cardiopatía isquémica entre ambos sexos (50 vs. $21,4 \%, \mathrm{p}=0,20)$.

Los pacientes que fallecieron presentaron la disnea como síntoma de ingreso más frecuente $(61,9 v s .69,6 \%)$, sin que se observaran diferencias. En cambio el síndrome confusional era el segundo síntoma más frecuente entre los pacientes fallecidos $(14,3 v s .4,1 \%)$ y la tos era menos frecuente que en el resto de pacientes estudiados $(14,3$ vs. $8,5 \%)$.

El análisis de las patologías crónicas mostró que el cumplimiento de los objetivos aconsejados tanto en la HTA $(33,3$ vs. $69,6 \%)$ como en la DM $(33,3$ vs. $41,6 \%, \mathrm{p}=0,62)$ no eran peores

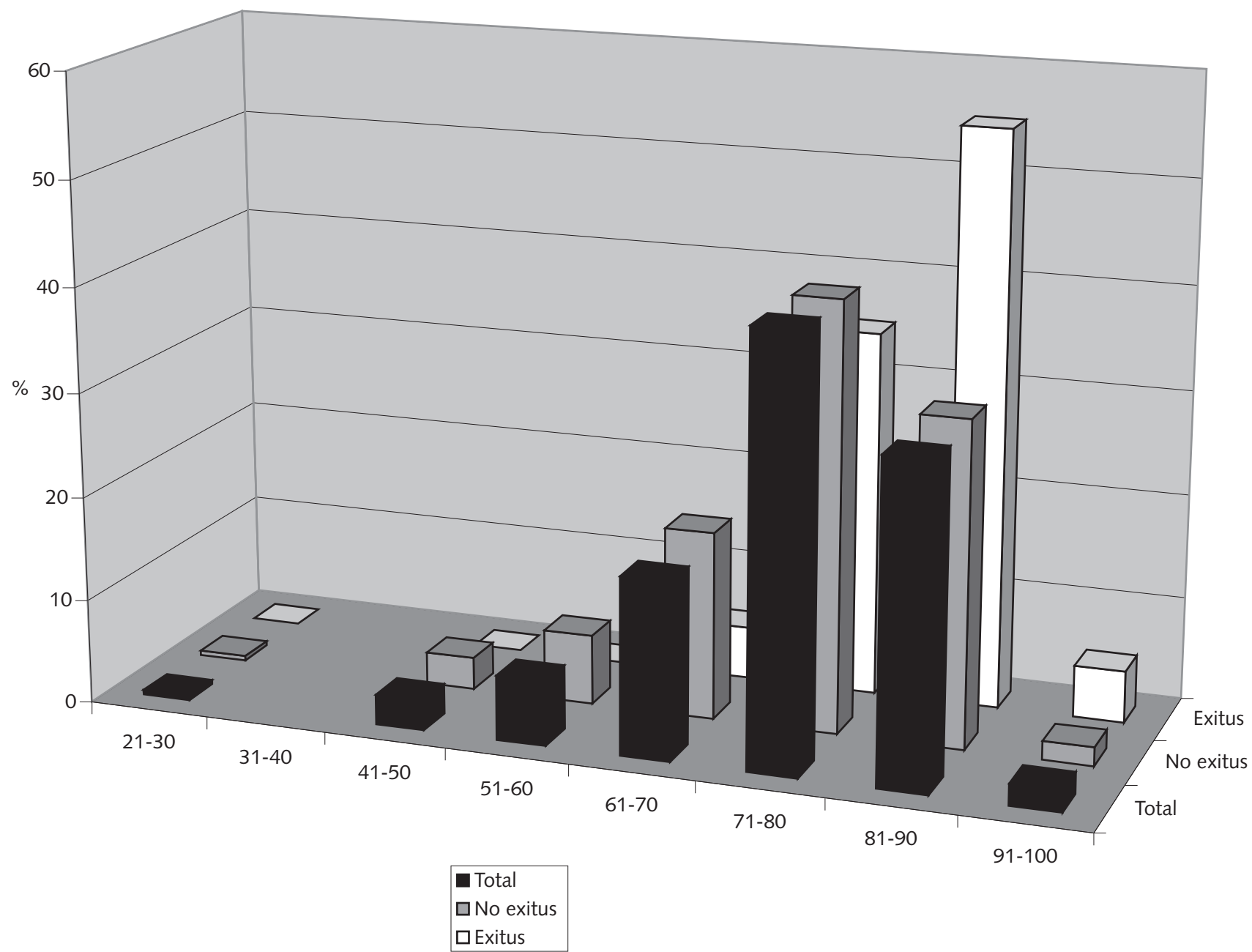

Fig. 1. Distribución por décadas de edad de los pacientes global, y por grupos de fallecidos/no fallecidos. 
entre pacientes fallecidos, $p=0,02$. El análisis cuantitativo de estos parámetros mostró cifras similares entre ambos grupos de presión arterial sistólica (PAS) (136 vs. $137 \mathrm{mmHg}$ ), $\mathrm{p}=0,91$, asimismo como de glucemia media (161 vs. $153 \mathrm{mg} / \mathrm{dl}), \mathrm{p}=0,93$.

Los ecocardiogramas realizados a los pacientes fallecidos $(23,8 \%)$ fueron similares al del resto de la población estudiada $(21,0 \%), p=0,76$. Entre los pacientes fallecidos, el porcentaje de pacientes con función sistólica deprimida fue superior $(80$ vs. $41,3 \%$ ), sin que se hallaran diferencias estadísticamente significativas, $\mathrm{p}=0,09$.

Entre las causas de descompensación cardíaca, el origen desconocido ocupa una de las causas más frecuentes $(28,6 \%)$ similar a la del resto de la población estudiada $(27,2 \%)$. Entre las causas conocidas, la infección respiratoria fue la más frecuente $(28,6 \%)$.

\section{DISCUSIÓN}

El presente estudio, como se ha comentado en las partes anteriormente publicadas*, ${ }^{*}$, presenta una población aunque de menor tamaño similar a las de otros estudios en cuanto a características epidemiológicas, tanto en estudios americanos (17), como europeos (18) o españoles (19).

En nuestro estudio se observó cierto predominio de sexo femenino, que podría explicarse por múltiples motivos, por un lado la mayor superviviencia de las mujeres y por otro la mayor prevalencia de HTA en mujeres y de cardiopatía isquémica en varones como causa de insuficiencia cardiaca, que condicionarían una mayor sobrecarga de presión en mujeres que originaría una mayor insuficiencia diastólica en este grupo (20) frente a una mayor insuficiencia sistólica en varones (21), por lo que se observaría una diferencia fisiológica que justificaría cierta diferencia en el pronóstico y mortalidad de los pacientes. En nuestro estudio no se observaron diferencias entre sexos en ninguno de estos parámetros, sin duda el reducido tamaño muestral podría tener un papel importante en esta limitación, sin embargo también es cierto que estudios de nuestro entorno, realizados también en ambiente hospitalario aportan datos similares, como el de Martínez-Sallez et al. (22) o, más recientemente el de Stella M. Macín et al. (23).

En nuestro estudio, hemos observado un predominio de la mortalidad en los pacientes de mayor edad, lo que es congruente con lo publicado por la mayor parte de los estudios realizados (24). En cambio estos estudios establecen cierta relación con un predominio del sexo femenino en pacientes de más edad (25), dato que también corroboramos en nuestro estudio aunque sin observar diferencias significativas, pero esto probablemente también se deba al limitado tamaño muestral.
El porcentaje de exitus presentado $(8,6 \%)$ es superior al recomendado por el Instituto Nacional de Salud (4\%) para la totalidad de población. Posiblemente los datos clínicos particulares de los pacientes con insuficiencia cardiaca (pluripatología), junto a su edad avanzada influyan de forma decisiva en esta elevada mortalidad (26).

El tiempo de ingreso medio de nuestros pacientes ha sido muy reducido (5 días), menor que la estancia media de la población global de estudio o en otros estudios similares (17-19) (entorno a 11 días). La mortalidad en las primeras 48 horas se asocia a factores extrahospitalarios, la elevada tasa de mortalidad en nuestros pacientes durante este periodo parece indicar que las patologías crónicas, fundamentalmente asociadas a insuficiencia cardíaca, que presentan los pacientes influyen en la mortalidad y no tanto los cuidados prestados durante el ingreso $(27,28)$.

Las infecciones respiratorias fueron la principal causa conocida de descompensación cardíaca entre los pacientes con IC, lo que es congruente con otros estudios realizados en un entorno similar (29).

Entre las limitaciones de este estudio, debemos comentar las mismas que para los otros trabajos presentados previamente*: diseño descriptivo observacional que limita el estudio de relaciones causales entre diversos factores epidemiológicos y la mortalidad y el reducido tamaño poblacional que ha dificultado la identificación de diferencias estadísticamente significativas. Ambas limitaciones colaboran en un posible sesgo de selección, sin embargo, creemos han sido superadas al emplear no una muestra sino una población (todos los pacientes ingresados a lo largo de 5 años por insuficiencia cardiaca) y, además, se ha comprobado como los datos epidemiológicos de nuestra población con otras mucho más amplias de nuestro entorno coinciden en todos los datos de relavancia (17-19). Una limitación más del estudio es la reducida tasa de ecocardiogramas realizados, comentado en los trabajos previamente publicados*,**, hecho que puede influir en la dificultad de observar diferencias estadísticamente significativas y valorables en la práctica clínica (por reducido tamaño poblacional: fallecimientos con ecocardiograma realizado) sobre la mayor o menor mortalidad de los pacientes en relación con la función sistólica.

Tras lo expuesto podemos concluir que: el único factor relacionado con la mortalidad es la edad avanzada de los pacientes. No podemos afirmar que ni sexo ni disfunción sistólica sean marcadores de riesgo de la mortalidad en los pacientes con insuficiencia cardiaca.

Como en los estudios previos, consideramos que sería necesario realizar un estudio longitudinal que permita identificar los factores asociados con la mortalidad.

\section{Bibliografía}

1. Boix R, Medrano MJ, Almazán J. Actualización de la mortalidad por enfermedades cardiovasculares arterioscleróticas: Enfermedad cardiovascular y enfermedad isquémica del corazón. Bol Epidemiol Sem 2000; 8: 77-80.

2. Levy D, Larson MG, Vasan RS, Kannel WB, Ho KKL. The progression from hypertension to congestive heart failure. JAMA 1996; 275: 1557-62

3. Cleland JGF, Gemmel I, Khand A, Boddy A. Is the prognosis of heart failure improving? Eur J Heart Fail 1999; 1: 229-241.

4. Barrios V, Ortega J. ACE inhibitors in the treatment of heart failure.
Heart Failure Train Lett 1999; 7: 2-5.

5. Poole-Wilson PA, Swedberg K, Cleland JGF, DiLenarda A, Hanrath P, Komadja M, et al, for the COMET Investigators. Comparison of carvedilol and metoprolol on clinical outcomes in patients with chronic heart failure in the Carvedilol or Metoprolol European Trial (COMET): Randomised controlled trial. Lancet 2003: 362: 7-13.

6. Cleland JGF, Khand A, Clark A. The heart failure epidemic: exactly how big is it? Eur Heart J 2001; 22: 623-6.

7. Rodríguez-Artalejo F, Banegas Banegas JR, Guayar-Castillón P. Epide- 
miología de la insuficiencia cardiaca. Rev Esp Cardiol 2004; 57 (2): 163-70.

8. Ho KL, Anderson KM, Kannel WB, Grossman W, Levy D. Survival after the onset of congestive heart failure in Framingham heart study subjects. Circulation 1993; 88: 107-15.

9. Schocken DD, Arrieta MI, Leaverton PE, Ross EA. Prevalence and mortality rate of congestive heart failure in the United States. J Am Col Cardiol 1992; 20: 301-6.

10. Cowburn PJ, Cleland JGF, Cotas AJS, Komajda M. Risk stratification in chronic heart failure. Eur Heart J 1998; 19: 696-710.

11. Adams KF Jr, Dunlap SH, Sueta CA, Clarke SW, Patterson JH, Blauwer $\mathrm{MB}$, et al. Relation between gender, etiology and survival in patients with sumptomatic heart failure. J Am Coll Cardiol 1996; 28: 1781-8.

12. Schindler DM, Kostis JB, Yusuf S, Quiñónez MA, Pitt B, Stewart D, et al. Diabetes mellitus, a predictor of morbidity and mortality in the Studies of Left Netricular Dysfunction (SOLVD). Trials and Registry. Am J Cardiol 1996; 77: 1017-20.

13. Varela-Román A, González-Juanatey JR, Basante P, Trillo R, GarcíaSeara J, Martínez-Sande JL, et al. Clinical characteristics and prognosis of hospitalizad inpatients with heart failure and preserved or reduced left ventricular ejection fraction. Heart 2002; 88: 249-54.

14. Braunwald E. The pathogenesis of the heart failure: then and now. Medicine 1991; 70: 68.

15. Joint National Committee on Prevention, Detection, Evaluation, and Treatment og High Blood Pressure. The Seventh report of the Joint National Committee on Prevention, Detection, Evaluation, and Treatment of High Blood Presure (JNC VII). JAMA 2003; 289: 2560-72.

16. Expert Committee on the diagnosis and classification of Diabetes Mellitus. American Diabetes Association: clinical practice recommendations 2002. Diabetes Care 2002; 25: (Supl. 1): S1-147.

17. Senni M, Tribouilloy CM, Rodeheffer RJ, JAcobsen SJ, Evans JM, Bailey KR, et al. Congestive Herat failure in the comunity. A study of all incident cases in Olmsted Country. Minnesonta in 1991. Circulation 1998; 98: 2282-9.

18. Cowle MR, Word DA, Cotas AJS, Thomson SG, Poole-Wilson PA, Suresh V, et al. Incidence and aetiology of heart failure: A populationbased study. Eur Heart J 1999; 20: 421-8.
19. Barrios Alonso et al. Hipertensión Arterial e Insuficiencia Cardiaca en las consultas de Atención Primaria y Cardiología de España. Rev Clin Esp 2003; 203 (7): 334-42.

20. Bech-Hanssen O, Wallentin I, Houltz E, Beckman Suurkula M, Larsson $\mathrm{S}$, Caidahl K. Gender differences in patients with severe aortic stenosis: Impact on preoperative left ventricular geometry and function, as well as early postoperative morbidity and mortality. Eur J Cardiothorac Surg. 1999; 15: 24-30.

21. Kannel WB, Belanger AJ. Epidemiology of Herat failure. Am Herat J. 1991; 121: 951-7.

22. Martínez-Sellés M, García Robles JA, Prieto L, Domínguez Munoa M, Frades E, Díaz-Castro O, et al. Systolic dysfunction is a predictor of long term mortality in men but not in women with Herat failure. Eur Heart J. 2003; 24: 2046-53.

23. Macín SM, Perna ER, Címbaro Canella JP, Alvarenga P, Pantich R, Ríos N, Farías EF, Bardasco JR. Características clinicoevolutivas en la insuficiencia cardíaca descompensada con disfunción sistólica y función sistólica preservada. Rev Esp Cardiol 2004; 57 (1): 45-52.

24. Remme W, Swedberg K. Guidelines for the diagnosis and treatment of chronic heart failure. Task Force for the diagnosis and treatment of chronic heart failure. European Society of Cardiology. Eur Heart J 2001; 22: 1527-1560.

25. Grossman W. Defining diastolic dysfunction. Circulation 2000; 101: 2020-1.

26. Gottdiener JS, Arnold AM, aurigemma G. Predictors of congestive heart failure in the elderly: the Cardiovascular Health Study. J am Coll Cardiol 2000; 35: 1628-37.

27. Beguin C, Boland B, Van Mullem X, Van Mullem T, France F, Coche E et al. Fiftheen years of in a patient care in an academic division of general internal medicine: 1980-1994. Trends over time in patient's diagnoses, case-severity, case-complexity and mortality rate. Acta Clin Belg 1996; 51: 311-9.

28. Longo DR, Bohr D. Métodos cuantitativos en la gestión de la calidad. Una guía práctica. Barcelona: SG editores, 1994.

29. Sánchez J, Gómez A, Amor J, García M, Campoy L, Pena P. Análisis of hospital mortality at a regional hospital. An Med Interna (Madrid) 1997; 14: 71-5. 